\title{
Evaluación de la calidad de suelos en agroecosistemas de Colombia a través de la selección de un conjunto mínimo de datos
}

\author{
Evaluation of soill quality in agroecosystems of Colombia \\ through the selection of a minimum data set
}

\author{
Laura Natali Afanador-Barajas ${ }^{1}$, Dainer Andrés Coca Peña ${ }^{(\mathbb{C})}$, Andrés Felipe Vargas Giraldo ${ }^{3}$, \\ María Fernanda Bautista Murcia ${ }^{\circledR}$, Angélica Mendoza Hernández ${ }^{5}$ \\ y Victoria Eugenia Vallejo Quintero ${ }^{* * \mathbb{E}}$
}

Afanador-Barajas, L.N., Coca-Peña, D.A., Vargas-Giraldo, A.P., Bautista-Murcia, M.F., Mendoza-Hernández, A. y Vallejo-Quintero, V.E. (2020). Evaluación de la calidad de suelos en agroecosistemas de Colombia a través de la selección de un conjunto mínimo de datos. Colombia forestal, 23(1), 35-50.

Recepción: 7 de mayo 2019

\section{Resumen}

El objetivo de esta investigación fue evaluar la calidad de suelos mediante índices de calidad constituidos por tres conjuntos mínimos de datos (tres, cinco y seis indicadores), en cinco agroecosistemas con cultivo de café (Coffea arabica) orgánico, guatila o chayote (Sechium edule), policultivo (Anthurium andraeanum, Manihot esculenta, Musa balbisiana o Zea mays), pastizal convencional (Pennisetum clandestinum y Cynodon dactylon) y Guaduales (Guadua angustifolia), en el municipio de Cachipay (Cundinamarca). Durante el muestreo se seleccionaron aleatoriamente dos áreas representativas para cada agroecosistema y se delimitaron dos cuadrantes $(2.5 \times 2.5 \mathrm{~m})$ de los que se tomaron dos muestras
Aprobación: 16 de diciembre 2019

compuestas de suelo (20 submuestras; profundidad de 0-15 cm). Se evidenció que independiente del conjunto mínimo de datos empleado, los menores índices de calidad de suelos lo presentaron el pastizal convencional y el cultivo de guatila. Los valores mayores del índice de calidad correspondieron al policultivo, guaduales y cultivo de café. Se concluye que el uso de variables como la densidad aparente, el índice de estabilidad, el pH, la actividad deshidrogenasa, el recuento de heterótrofos y las bacterias solubilizadoras de fosfato son indicadores relevantes que permiten evaluar adecuadamente la calidad edáfica en agroecosistemas.

Palabras clave: agroecología, Cachipay, degradación del suelo, índices de calidad, propiedades del suelo.

1 Grupo de investigación: Biomat. Universidad Central. Facultad de Ingeniería y Ciencias Básicas. Universidad Central. lafanadorb@ucentral.edu.co.

2 Grupo de investigación: Agua y Desarrollo Sostenible. Facultad de Ingeniería y Ciencias. Básicas. Universidad Central. dcocap@ucentral.edu.co.

3 Grupo de investigación: Agua y Desarrollo Sostenible. Facultad de Ingeniería y Ciencias Básicas. Universidad Central. avargasg4@ucentral.edu.co.

4 Grupo de investigación: Agua y Desarrollo Sostenible. Facultad de Ingeniería y Ciencias Básicas. Universidad Central. mbautistam1@ucentral.edu.co.

5 Grupo de investigación: Agua y Desarrollo Sostenible. Facultad de Ingeniería y Ciencias Básicas. Universidad Central. amendozah@ucentral.edu.co.

6 Grupo de investigación: Agua y Desarrollo Sostenible. Facultad de Ingeniería y Ciencias Básicas. Universidad Central

* Autor de correspondencia: evallejoq@ucentral.edu.co. 


\begin{abstract}
This study aimed to evaluate the soil quality through quality indexes consisting of three minimum data sets (3, 5 and 6 indicators) in five agroecosystems with organic coffee (Coffea arabica), guatila or chayote (Sechium edule), polyculture (Anthurium andraeanum, Manihot esculenta, Musa balbisiana o Zea mays), conventional pasture (Pennisetum clandestinum y Cynodon dactylon) and Guadua (Guadua angustifolia), in the municipality of Cachipay (Cundinamarca). During the sampling, two representative areas were randomly selected for each agroecosystem and two quadrants $(2.5 \times 2.5 \mathrm{~m})$ were delimited, from which two composite samples of
\end{abstract}

soil were taken (20 subsamples, depth of $0-15 \mathrm{~cm}$ ). It was evidenced that independent of the minimum set of data used, the lowest soil quality indexes were presented by the conventional pasture and the guatila culture. The highest values of the quality index corresponded to polyculture, forest and organic coffee cultivation. In conclusion, the use of variables such as bulk density, stability index, $\mathrm{pH}$, dehydrogenase activity, heterotrophic density and solubilized phosphate bacteria are important indicators to measure the soil quality in agroecosystems.

Keywords: Agroecology, Cachipay, soil quality index. soil properties, soil degradation.

\section{INTRODUCCIÓN}

El suelo es considerado un recurso natural no renovable, fundamental para los humanos y la biósfera en general. Su relevancia radica en que provee servicios ecosistémicos esenciales para el equilibrio terrestre. Por lo anterior, la conservación y mantenimiento de la calidad del suelo es fundamental para la seguridad alimenticia, la productividad agropecuaria y la sostenibilidad ecológica (Yu et al., 2018). Doran y Parkin definieron la calidad del suelo como: "la capacidad del suelo para funcionar dentro de los límites del ecosistema, para atenuar los contaminantes ambientales y patógenos, sostener la productividad de los animales y plantas, y sostener la salud humana" (1994, p. 3). Este concepto se ha utilizado como herramienta para diagnosticar el estado general de los suelos, siendo útil en la toma de decisiones sobre alternativas de uso y manejo agropecuario que logren minimizar los procesos degradativos edáficos y favorezcan el mejoramiento de la calidad actual del suelo.

El suelo puede comprometer su salud o calidad, cuando se enfrenta a diversos cambios en su uso o se aplican prácticas de manejo agropecuario inapropiadas. Estos usos pueden alterar la regulación natural de los agroecosistemas, convirtiéndolos en sistemas con baja resiliencia y altamente dependientes de insumos químicos externos y de la intervención humana (Vallejo, 2013; Mursec et al., 2018). Así, la evaluación, valoración y monitoreo de la calidad edáfica en distintos agroecosistemas es fundamental para evaluar el manejo sostenible del suelo (Obade y Lal, 2016; Guo et al., 2017).

La calidad edáfica abarca los componentes físicos, químicos y biológicos del suelo y sus interacciones; por lo cual es necesario evaluarlos de manera conjunta. Dichas variables constituyen los indicadores de calidad del suelo (IC) puesto que representan una condición e indican cambios o tendencias de esa condición, convirtiéndose en herramientas de medición que ofrecen información sobre las propiedades, procesos y características del suelo (Ghaemi et al., 2014). Sin embargo, no todos los parámetros tienen la misma relevancia para todos los suelos o situaciones (p. ej., tipo de suelo, topografía, condiciones de edafogénesis, actividades antrópicas y condiciones climáticas). Además, existe subjetividad al asignar peso a cada indicador para la elaboración o empleo de índices propuestos y el establecimiento de sus indicadores (líneas de base y umbrales críticos) (Wilson, 2017), por lo cual, no existen IC universales que sean 
adecuados, sensibles y comprensibles en diversos propósitos y contextos (Cantú, Becker, Bedano y Schiavo, 2007; García, Ramírez y Sánchez, 2012; Estrada- Herrera, 2017). De acuerdo con esto, la selección adecuada de los IC requiere ser planteada teniendo en cuenta las condiciones particulares de cada región.

Debido a la complejidad para evaluar la calidad edáfica y los inconvenientes a la hora de seleccionar un IC, se ha sugerido identificar un número adecuado de propiedades edáficas que determinen y representen los principales procesos y funciones que ocurren en suelo, lo que se conoce como conjunto mínimo de datos (CMD). Con lo cual, además de ahorrar tiempo y dinero, se obtiene una estimación confiable y precisa de la calidad edáfica en agroecosistemas (Zhang et al., 2016). Los primeros CMD se basaron en el juicio de los expertos (Doran y Parkin, 1994), posteriormente, se ha empleado una variedad de métodos estadísticos multivariados para seleccionar los IC e integrarlos en índices de calidad de suelos (ICS) con el objeto de valorar el impacto de distintos sistemas agrícolas (Masto et al., 2007; Muñoz-Rojas, 2018). Los ICS se definen como un "conjunto de parámetros que, cuando se interrelacionan, proporcionan datos numéricos sobre la capacidad de un suelo para realizar sus funciones; resumiendo, en un único valor, un grupo de variadas y complejas propiedades que representan las características del suelo" para autores como Andrews, Karlen y Mitchell (2002), Navas, Benito, Rodríguez y Masaguer (2011) y Muñoz-Rojas (2018). Los pasos principales para calcular el ICS son: 1) seleccionar el CMD de los indicadores; 2) transformar los indicadores a puntuaciones; y 3) combinar o integrar los puntajes en el ICS.

Estudios previos en la zona realizados por Vallejo et al., (2018) mostraron un deterioro en la calidad edáfica del pastizal convencional (PA) Por el contrario, el policultivo (PO) y café (CA) presentaron una mejoría en la mayoría de las propiedades fisicoquímicas edáficas, lo cual tuvo efecto positivo en la densidad y actividad microbiana. El objetivo del presente estudio fue evaluar la calidad edáfica en cinco agroecosistemas del municipio de Cachipay, Cundinamarca (Colombia), mediante tres CMD, seleccionados y determinados de herramientas multivariadas, en este caso, el análisis de componentes principales (ACP). A partir del presente trabajo se seleccionaron indicadores (físicos, químicos y microbiológicos) de procesos fundamentales del suelo que se incorporaron en índices de calidad, constituyéndose en herramientas idóneas para la evaluación y monitoreo de la calidad edáfica (escala regional) en agroecosistemas colombianos. Esto, en conjunto con la interacción y aprovechamiento del conocimiento de los agricultores, contribuirá a la comprensión de los factores que influyen las distintas prácticas de manejo agrícola en los sistemas seleccionados y su efecto sobre distintos componentes del suelo, con el fin de lograr una adecuada selección, mejoría y diseño de agroecosistemas sostenibles.

\section{MATERIALES Y MÉTODOS}

\section{Área de estudio}

El área de estudio fue el campo experimental de tecnologías ambientales, localizado en la casa de eetiro y encuentros Nazareth de la comunidad religiosa Hermanas de la Caridad Dominicas de la Presentación de la Santísima Virgen, en la vereda Petaluma (4. ${ }^{\circ} 44^{\prime} 53.34^{\prime \prime}$ latitud norte, $74 .^{\circ} 25^{\prime}$ $32.14^{\prime \prime}$ longitud oeste). El régimen de Iluvias es bimodal, con dos periodos de precipitación (abril y octubre) y dos periodos secos (enero y junio). La precipitación promedio anual es de $1492 \mathrm{~mm}$ y la temperatura oscila entre los 16.1 a $27.7{ }^{\circ} \mathrm{C}$. La finca tiene un área de 10.06 ha y cuenta con un relieve montañoso en el que su vegetación corresponde a guaduales. El uso actual del suelo en el municipio es ganadería extensiva con pasto Pennisetum clandestinum y explotación de cultivos de papa, mora, flores, café y frutales. Las texturas de sus suelos son moderadamente gruesas 
(franco-limosas y franco-arenosas) (EOT Cachipay, 2008; Vallejo et al., 2018).

\section{Muestreo del suelo}

Para el muestreo de suelo se seleccionaron dos áreas independientes para cada uno de los cinco agroecosistemas escogidos: con cultivo de café (Coffea arabica) orgánico (CA), guatila o chayote (Sechium edule; GU), policultivo (Anthurium andraeanum, Manihot esculenta, Musa balbisiana o Zea mays; PO), pastizal convencional (Pennisetum clandestinum y Cynodon dactylon; PA) y guadual (Guadua angustifolia; GA). El policultivo (PO) y café (CA) reciben en su mayoría fertilización orgánica con residuos agrícolas cada tres y seis meses, respectivamente; del mismo modo, la labranza es manual. El cultivo de guatila (GU) recibe fertilización con gallinaza cada ocho meses y labranza manual. El pastizal (PA) había recibido un manejo convencional con uso de glifosato, fertilización química nitrogenada y labranza con tractor; en la actualidad se encuentra en etapa de descanso. Por último, en el guadual (GA) no se realiza ningún de intervención antropogénica. Para más detalle de los manejos en los agroecosistemas consúltese Vallejo et al. (2018). En cada agroecosistema se establecieron dos cuadrantes $(2.5 \times 2.5 \mathrm{~m})$, en los cuales se tomaron con barrenos metálicos dos muestras compuestas aleatorias constituidas a partir de 20 submuestras $(0-15 \mathrm{~cm}$ de profundidad). Cada muestra de suelo se almacenó en bolsas de plástico herméticas debidamente rotuladas hasta su Ilegada al laboratorio de suelos (Universidad Central). Las muestras se dividieron en dos porciones: una parte fue tamizada (2 mm) y secada a temperatura ambiente, para análisis fisicoquímicos y la otra parte fue refrigerada $\left(4^{\circ} \mathrm{C}\right)$ para la realización de análisis microbiológicos y actividades enzimáticas.

\section{Análisis fisicoquímicos y microbiológicos}

Los métodos utilizados para determinar la mayoría de las propiedades fisicoquímicas y microbiológicas: diámetro ponderado medio (DPM), diámetro geométrico medio (DGM), índice de estabilidad (IE), humedad $(\mathrm{H}), \mathrm{pH}$, textura, carbono orgánico $(\mathrm{C})$, actividad de catalasa (CAT) y recuento de heterótrofos $(\mathrm{HT})$, se realizaron según metodologías descritas por Vallejo et al. (2018). Adicionalmente, para esta investigación se incluyeron otras variables como la densidad real (DR), densidad aparente (DA) y porosidad (POR) (Flores y Alcalá, 2010), actividad deshidrogenasa (ADH) (Casida, Klein y Santoro, 1964), recuento de actinomicetos (ACT) (Goodfellow y Williams, 1983) y bacterias solubilizadoras de fosfato (BSF) (Nautiyal, 1999).

\section{Definición y selección del conjunto mínimo de datos (CMD)}

El primer paso consistió en realizar un análisis de varianza (Andeva), teniendo en cuenta el conjunto total de variables (16) fisicoquímicas y microbiológicas. Esta fase permitió excluir las propiedades que no presentaron diferencias estadísticamente significativas en respuesta a los diferentes manejos agrícolas en los agroecosistemas evaluados. Lo anterior se realizó con el programa SPSS Statistics $^{\circledR} 242010$ (v. 24, Chicago, IL). Solo los indicadores que mostraron diferencias estadísticamente significativas entre tratamientos $(p \leq 0.05)$ se seleccionaron para la matriz de datos total preliminar y determinar el CMD (Raiesi y Salek-Gilani, 2018). Seguidamente, se efectuó un análisis de similitud en una vía (Anosim —analysis of similarities por sus siglas en inglés-), esta corresponde a una prueba no paramétrica que permite determinar diferencias significativas entre dos o más grupos, basada en la medida de cualquier distancia (Ramette, 2007). En este caso, se determinó la similitud entre las distintas de las muestras de suelo para cada agroecosistema (grupos) a partir de las distancias de Bray-Curtis con una permutación de 9999. Para lo anterior, se empleó el programa estadístico Paleontological Statistics (PAST), versión 2.16 (Hammer, Harper y Ryan, 2012). El programa 
estadístico calculó el valor $\mathrm{R}$, el cual denota disimilitud entre grupos si su valor se aproxima o sobrepasa a 1. La significancia estadística de dicho coeficiente se obtuvo mediante un proceso de aleatorización, y se optó por un valor referencial: $\mathrm{p} \leq 0.05$; valores de $\mathrm{R} \leq 0.50$ indicaron una débil separación entre los grupos comparados (Ramette, 2007). Los indicadores físicos, químicos y microbiológicos que presentaron diferencias significativas (Andeva, $\mathrm{p} \leq 0.05$ ) se sometieron a un ACP empleando una matriz de datos estandarizada (matriz de correlación) con el paquete estadístico FactoMineR (Lê, Josse y Husson, 2008) en el programa R (R Development Core Team, 2016).

El ACP fue la herramienta empleada para reducir el grupo o conjunto de variables originales, la cual facilitó la selección de los indicadores más apropiados para evaluar y valorar la calidad edáfica en los agroecosistemas comparados; eliminando aquellas variables que aportaban poco al estudio. Para la construcción del CMD, solo se seleccionaron los componentes principales (CP) que tuvieron valores propios (eigenvalues) $\geq 1$ y aqueIlos que explicaron al menos el $5 \%$ de la variación total del conjunto total de datos. Los CP fueron evaluados basándose en los valores de carga de las variables individuales (la correlación entre la variable y el componente principal). Se escogieron tres CMD, el primero seleccionó solo aquellos indicadores con la mayor correlación en los CPs con valores propios $\geq 1$, el segundo CMD se constituyó con los indicadores que tuvieran una correlación $\geq 0.75$ (Rezaei, Gilkes y Andrews, 2006; Ghaemi et al., 2014), y para el tercero solo las propiedades altamente cargadas con un valor de peso mayor o igual $10 \%$ de la mayor carga ponderada del respectivo componente fueron considerados como indicadores pertinentes para la indexación dentro de ese CP y selección del CMD. Cuando más de un indicador fue retenido en cada $\mathrm{CP}$, la redundancia del indicador fue evaluada utilizando la correlación de Pearson (Navas et al., 2011; Yu et al., 2018).

\section{Cálculo del ICS}

Después de obtener los tres CMD, se procedió a asignar una categoría a cada uno de los indicadores seleccionados, en el cual un valor alto se consideró como "bueno" o "malo" en términos de la propiedad y su función en el suelo. Se evitaron las funciones de puntuación no lineal debido a su menor capacidad para pronosticar la variable del punto final o el rendimiento del cultivo (Andrews et al., 2002). Los parámetros del suelo se dividieron en grupos de acuerdo con tres funciones: a) "más es mejor" (p. ej., DPM, IE, H, C, ADH, HT); b) "menos es mejor" (p. ej., DA); y (c) "óptimo" (p. ej., pH). Las propiedades "óptimas" son aquellas que tienen una influencia positiva hasta un cierto nivel más allá del cual la influencia podría considerarse perjudicial (Fernandes et al., 2011). En este caso un $\mathrm{pH}$ entre 5.5-7.2 fue punteado con 1, menor a 5.5 fue clasificado como "más es mejor" y mayor de 7.2 fue clasificado como "menos es mejor". Para los parámetros 'más es mejor', cada observación se dividió por el valor más alto observado de todo el conjunto de datos, de modo que el valor más alto observado tendría una puntuación de 1; para los parámetros "menos es mejor", el valor observado más bajo en todo el conjunto de datos se dividió por cada observación, de modo que el valor observado más bajo recibió una puntuación de 1; y los parámetros "óptimos" se calificaron hasta un valor umbral ya que "más es mejor", y después de eso, los valores umbrales se puntuaron como "menos es mejor" (Wymore, 1993; Andrews et al., 2002; Mandal et al., 2008).

El índice de calidad del suelo (ICS) se usó para evaluar la calidad del suelo en los diferentes sistemas productivos (SP). La técnica de indexación utilizada es ICS (1) aditivo ponderado propuesto por Karlen y Stott (1994), el cual ha sido ampliamente utilizado (Fernandes et al., 2011; Chen et al., 2013; Nabiollahi et al., 2017; Yu et al., 2018). Las calificaciones previamente 
establecidas para todos los indicadores se integraron en la siguiente ecuación:

$$
\text { (1) } I C S=\sum_{1}^{n} W i S i
$$

Donde $\mathrm{W}$ es el factor de peso dado por la variabilidad del CP correspondiente al indicador seleccionado sobre el total de la variación y $\mathrm{S}$ es la puntuación asignada a cada indicador, para establecer el índice de calidad de suelo correspondiente a todo el lote experimental. Cada CP explica cierto porcentaje de la variación en el CMD; este porcentaje es dividido por el total de la variación explicada en el CP seccionado para ese indicador en el CMD (si la variación de un CP usaba varios indicadores, ese valor se dividió por el número total de indicadores correspondientes), dando así el factor de peso (W), dependiendo del número de indicadores en el CMD, peso asignado cambiaba (tabla 1), también se muestra el promedio de puntuación asignado para cada indicador. Para ver las diferencias de los ICS calculados entre sistemas productivos (SP) se realizó un análisis de varianza (Andeva, $\mathrm{p} \leq 0.05$ ) y para evaluar si existían diferencias entre las variables en los diferentes agroecosistemas y en los tres CMD seleccionados se realizó una prueba de kruskal wallis $(\mathrm{p} \leq 0.05)$, usando programa $\mathrm{R}$ ( $\mathrm{R}$ Development Core Team, 2016).

\section{RESULTADOS}

\section{Selección de los indicadores para el CMD}

En el presente estudio fueron medidas 16 propiedades edáficas como potenciales indicadores de calidad de suelos en los agroecosistemas. Se evidenciaron diferencias estadísticamente significativas (Andeva, $\mathrm{p} \leq 0.05$ ) en la mayoría de las variables fisicoquímicas y microbiológicas, excepto la densidad real, la porosidad, la textura, la resistencia a la penetración y la estabilidad de agregados (Andeva, $\mathrm{p} \geq 0.05$ ). Por este motivo, únicamente 12 variables fueron incluidas en la matriz final de datos (tabla 2) para ser evaluadas a través del Anosim y ACP para la selección del $C M D$, para reducir, de esta manera, la redundancia de indicadores para el cálculo del ICS. Por otra parte, se presentaron diferencias estadísticamente significativas (Kruskal wallis, $\mathrm{p} \leq 0.05$ ) entre los agroecosistemas evaluados para las variables IE, pH, C, ADH, HT, BSF y ACT (tabla 2).

Se evidenció un efecto significativo del manejo agrícola en los agroecosistemas sobre las propiedades edáficas, lo que permitió corroborar que las variables incluidas en esa matriz de datos explicaban adecuadamente la variabilidad total de los mismos y, de esta manera, exponían con claridad las diferencias entre los agroecosistemas (figura 1) (Anosim, R global=0.72; $\mathrm{p}<0.0001$ ). Así mismo,

Tabla 1. Valores de factor de peso (W) según la variación en el análisis de CP y la puntuación (S) utilizados para cada uno de los indicadores seleccionados en los CMD. IE: índice de estabilidad; BSF: bacterias solubilizadoras de fosfato; DA: densidad aparente; HT: recuento de heterótrofos totales; y ADH: actividad de deshidrogenasa

\begin{tabular}{ccccc}
\hline Indicador & W (CMD 3) & W (CMD 5) & W (CMD6) & S (promedio) \\
\hline IE & & 0.20 & 0.15 & 0.70 \\
DA & & 0.20 & 0.15 & 0.75 \\
pH & 0.61 & 0.20 & 0.15 & 0.89 \\
ADH & 0.15 & 0.19 & 0.15 & 0.67 \\
HT & 0.24 & & 0.24 & 0.81 \\
BSF & & 0.20 & 0.15 & 0.97 \\
\hline
\end{tabular}


Tabla 2. Promedio de las variables utilizadas en los diferentes agroecosistemas $(n=4)$. $P A=$ pastizal, $G U=$ cultivo de guatila, $\mathrm{PO}=$ policultivo, $\mathrm{CA}=$ cultivo de café orgánico y $\mathrm{GA}=$ guaduales. IE: índice de estabilidad; BSF: bacterias solubilizadoras de fosfato; DA: densidad aparente; HT: recuento de heterótrofos totales; C: carbono orgánico del suelo; DGM: diámetro geométrico medio; DPM: diámetro ponderado medio; $\mathrm{H}$ : humedad; ADH: actividad de deshidrogenasa y ACT: recuento de actinomicetos

\begin{tabular}{cccccc}
\hline Indicador & PO & CA & PA & GU & GA \\
\hline IE & $6.05 \pm 1.81 \mathrm{~b}$ & $6.55 \pm 1.42 \mathrm{~b}$ & $7.73 \pm 0.66 \mathrm{a}$ & $7.28 \pm 1.04 \mathrm{ab}$ & $8.9 \pm 1.07 \mathrm{ab}$ \\
DPM & $1.78 \pm 0.18 \mathrm{a}$ & $1.99 \pm 0.28 \mathrm{a}$ & $2.43 \pm 0.40 \mathrm{a}$ & $2.07 \pm 0.25 \mathrm{a}$ & $2.35 \pm 0.08 \mathrm{a}$ \\
$\mathrm{DGM}$ & $0.31 \pm 0.16 \mathrm{a}$ & $0.48 \pm 0.21 \mathrm{a}$ & $0.80 \pm 0.30 \mathrm{a}$ & $0.52 \pm 0.20 \mathrm{a}$ & $0.63 \pm 0.10 \mathrm{a}$ \\
$\mathrm{H}$ & $32.81 \pm 4.22 \mathrm{a}$ & $29.32 \pm 5.38 \mathrm{a}$ & $24.42 \pm 3.14 \mathrm{~b}$ & $31.14 \pm 1.12 \mathrm{a}$ & $31.04 \pm 1.43 \mathrm{a}$ \\
$\mathrm{DA}$ & $0.64 \pm 0.07 \mathrm{a}$ & $0.76 \pm 0.08 \mathrm{a}$ & $0.83 \pm 0.01 \mathrm{a}$ & $0.82 \pm 0.06 \mathrm{a}$ & $0.78 \pm 0.08 \mathrm{a}$ \\
PH & $6.01 \pm 0.50 \mathrm{a}$ & $5.59 \pm 0.12 \mathrm{ab}$ & $4.66 \pm 0.18 \mathrm{~b}$ & $5.58 \pm 0.36 \mathrm{ab}$ & $5.50 \pm 0.22 \mathrm{ab}$ \\
$\mathrm{C}$ & $7.13 \pm 0.92 \mathrm{a}$ & $6.59 \pm 0.99 \mathrm{a}$ & $2.64 \pm 0.52 \mathrm{~b}$ & $3.30 \pm 0.46 \mathrm{~b}$ & $2.49 \pm 0.54 \mathrm{~b}$ \\
$\mathrm{ADH}$ & $0.30 \pm 0.06 \mathrm{~b}$ & $0.31 \pm 0.02 \mathrm{ab}$ & $0.40 \pm 0.02 \mathrm{ab}$ & $0.47 \pm 0.03 \mathrm{a}$ & $0.31 \pm 0.06 \mathrm{~b}$ \\
HT & $7.77 \pm 0.28 \mathrm{ab}$ & $6.77 \pm 0.16 \mathrm{~b}$ & $5.94 \pm 0.17 \mathrm{~b}$ & $7.82 \pm 0.16 \mathrm{ab}$ & $8.88 \pm 0.20 \mathrm{a}$ \\
$\mathrm{BSF}$ & $7.06 \pm 0.05 \mathrm{ab}$ & $7.08 \pm 0.05 \mathrm{a}$ & $7.08 \pm 0.04 \mathrm{~b}$ & $7.10 \pm 0.07 \mathrm{ab}$ & $7.12 \pm 0.08 \mathrm{a}$ \\
ACT & $4.84 \pm 0.11 \mathrm{a}$ & $4.99 \pm 0.29 \mathrm{a}$ & $5.11 \pm 0.37 \mathrm{ab}$ & $5.23 \pm 0.34 \mathrm{~b}$ & $5.41 \pm 0.06 \mathrm{~b}$ \\
\hline
\end{tabular}

Media \pm desviación estándar, letras diferentes muestran diferencias significativas entre los agroecosistemas evaluados $(p<\leq 0.05$, Kruskal Wallis).

a través de la gráfica de ordenación (figura 1) se identificó claramente la separación entre los grupos comparados (agroecosistemas), mostrando una mayor disimilitud entre el PA y el resto de los agroecosistemas, particularmente, con el PO y GA (Anosim, $\mathrm{R}=1 ; \mathrm{R}=0.98 ; \mathrm{p}<0.0001$ ).

Los tres primeros CP presentaron valores propios $>1$ y explicaron el $75 \%$ de la varianza total

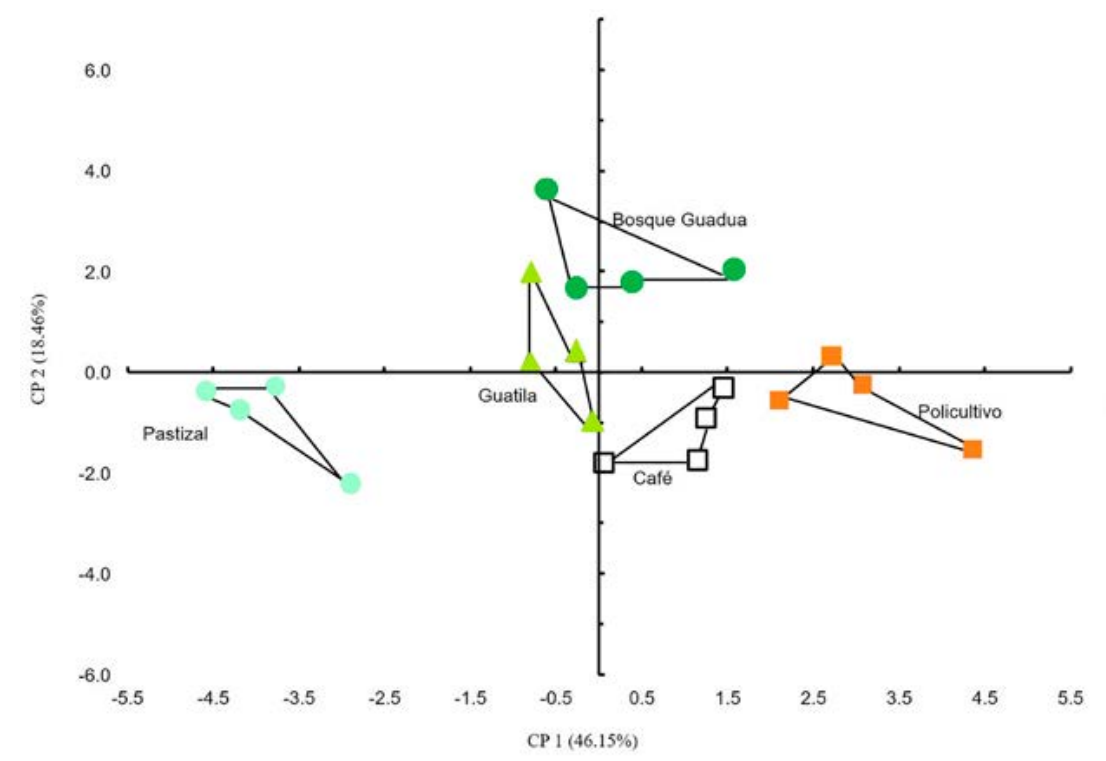

Figura 1. ACP de las variables fisicoquímicas y biológicas evaluadas para los diferentes agroecosistemas $(n=4)$. Se muestra entre paréntesis el porcentaje de varianza explicada para cada CP. 
de los datos. El primero explicó el $46 \%$ de la variación, el segundo el 17 \% y el tercero el $12 \%$; de la misma forma, los valores propios disminuyeron del CP 1 al CP 3 (tabla 3). Los resultados indican que los tres primeros $\mathrm{CP}$ expresaron efectivamente los cambios totales de la calidad de suelo en los agroecosistemas comparados. Los CMD se conformaron por las siguientes variables: para el primer CMD de seis variables: DA, IE, $\mathrm{pH}, \mathrm{ADH}, \mathrm{HT}$ y BSF; el segundo grupo se constituyó por cinco variables: DA, IE, $\mathrm{pH}, \mathrm{ADH}$ y $\mathrm{BSF}$; y para el tercer y último $\mathrm{CMD}$ se tuvieron en cuenta el $\mathrm{pH}$, la $\mathrm{ADH}$ y los HT. Es importante resaltar que independiente del CMD las variables que siempre se conservaron fueron el $\mathrm{pH}$ y la $\mathrm{ADH}$ (tabla 3).

\section{Evaluación de la calidad del suelo a través de índices de calidad}

Una vez que se seleccionaron y puntuaron, o calificaron los indicadores, se procedió a calcular
Ios ICS para todos los agroecosistemas evaluados (tabla 4). De manera general, se puede evidenciar que independiente del CMD seleccionado para la construcción del ICS, el PA presentó los menores valores de calidad en comparación con los otros agroecosistemas evaluados en el estudio. No obstante, solo para el ICS constituido con tres indicadores la diferencia fue significativa (tabla 4) y para el caso del CMD de cinco indicadores, también el cultivo de GU presentó bajos valores de calidad. Por su parte, los mayores valores de calidad obtenidos a través de los índices calculados muestran que para el caso del CMD constituido por cinco y seis indicadores los valores más altos los presentaron el $\mathrm{GA}>\mathrm{PO}>\mathrm{CA}>\mathrm{GU}$. Sin embargo, los ICS provenientes del $\mathrm{CMD}$ de tres indicadores la tendencia fue diferente, siendo $\mathrm{GU}>\mathrm{GA}=\mathrm{PO}>\mathrm{CA}$.

Letras minúsculas diferentes en cada columna indican diferencias significativas en los ICS entre los distintos agroecosistemas evaluados (Anova $\mathrm{p}<0.05$; Tukey). Letras mayúsculas diferentes

Tabla 3. Resultados del ACP realizado sobre las variables seleccionadas con el Anova. IE: índice de estabilidad; BSF: bacterias solubilizadoras de fosfato; DA: densidad aparente; HT: recuento de heterótrofos totales; C: carbono orgánico del suelo; DGM: diámetro geométrico medio; DPM: diámetro ponderado medio; H: humedad; ADH: actividad de deshidrogenasa y ACT: recuento de actinomicetos. Los parámetros con + indican los mayores autovectores o valores de carga generados por cada CP.

\begin{tabular}{cccc}
\hline & CP 1 & CP 2 & CP 3 \\
\hline Valores propios & 5.54 & 2.22 & 1.29 \\
\% Varianza & $46.15 \%$ & $18.46 \%$ & $11 \%$ \\
Varianza acumulada & & $75.6 \%$ & \\
\hline & Propiedades del suelo-valores de carga en cada CP & -0.170 \\
\hline pH & $0.877+$ & 0.113 & 0.325 \\
IE & $-0.825+$ & 0.119 & -0.010 \\
BSF & $0.774+$ & 0.366 & -0.162 \\
DA & $-0.765+$ & 0.130 & 0.335 \\
HT & 0.509 & $0.728+$ & 0.070 \\
C & 0.710 & -0.479 & 0.239 \\
DGM & -0.705 & 0.422 & 0.265 \\
DPM & -0.661 & 0.542 & -0.229 \\
H & 0.625 & 0.417 & $-0.791+$ \\
ADH & -0.526 & -0.010 & 0.309 \\
ACT & 0.189 & -0.658 & \\
\hline
\end{tabular}


Tabla 4. ICS calculados con los tres CMD construidos (3, 5 y 6 indicadores) en los diferentes agroecosistemas $(n=4) . P A=$ pastizal; $G U=$ cultivo de guatila, $P O=$ policultivo, $C A=$ cultivo de café orgánico y $G A=$ guaduales

\begin{tabular}{cccc}
\hline Agroecosistemas & CMD (3) & CMD (5) & CMD (6) \\
PO & $0.88 \mathrm{a} \mathrm{A}$ & $0.83 \mathrm{a} \mathrm{A}$ & $0.83 \mathrm{a} \mathrm{A}$ \\
$\mathrm{CA}$ & $0.86 \mathrm{a} \mathrm{A}$ & $0.80 \mathrm{a} \mathrm{A}$ & $0.79 \mathrm{ab} \mathrm{A}$ \\
PA & $0.71 \mathrm{~b} \mathrm{~B}$ & $0.77 \mathrm{~b} \mathrm{~A}$ & $0.74 \mathrm{~b} \mathrm{AB}$ \\
$\mathrm{GU}$ & $0.90 \mathrm{a} \mathrm{A}$ & $0.74 \mathrm{~b} \mathrm{~B}$ & $0.77 \mathrm{ab} \mathrm{AB}$ \\
$\mathrm{GA}$ & $0.88 \mathrm{a} \mathrm{A}$ & $0.84 \mathrm{a} \mathrm{A}$ & $0.87 \mathrm{a} \mathrm{A}$ \\
\hline
\end{tabular}

en cada fila indican diferencias significativas en los ICS para cada agroecosistema para cada CMD analizado (Kruskall-Wallis $\mathrm{p}<0.05$; bonferrini).

\section{Contribución de los indicadores al índice de calidad de suelos}

El porcentaje de contribución de los indicadores en la formación del ICS en los diferentes agroecosistemas se presentó a través de una gráfica radial, usando los tres CMD (figura 2). Para el CMD de tres indicadores se evidenció que el pH tuvo la mayor contribución (60-40 \%) en la diferenciación entre los SP, seguido de los HT con el $20 \%$, y la menor contribución para la actividad de la ADH. Mientras que en el CMD de cinco y seis indicadores se observaron diferencias de la contribución en los diferentes agroecosistemas, para cinco indicadores: $\mathrm{BSF}(18-10 \%)>\mathrm{pH}(14-19 \%)>\mathrm{DA}(13-18 \%)$ $>$ IE $(10-19 \%)>A D H(9-14 \%)$, y para seis indicadores: HT $(15-23 \%)>\operatorname{BSF}(14-15 \%)>\mathrm{pH}(11-13$ $\%)>$ DA $(10-13 \%)>$ IE $(8-14 \%)>\operatorname{ADH}(7-11 \%)$.

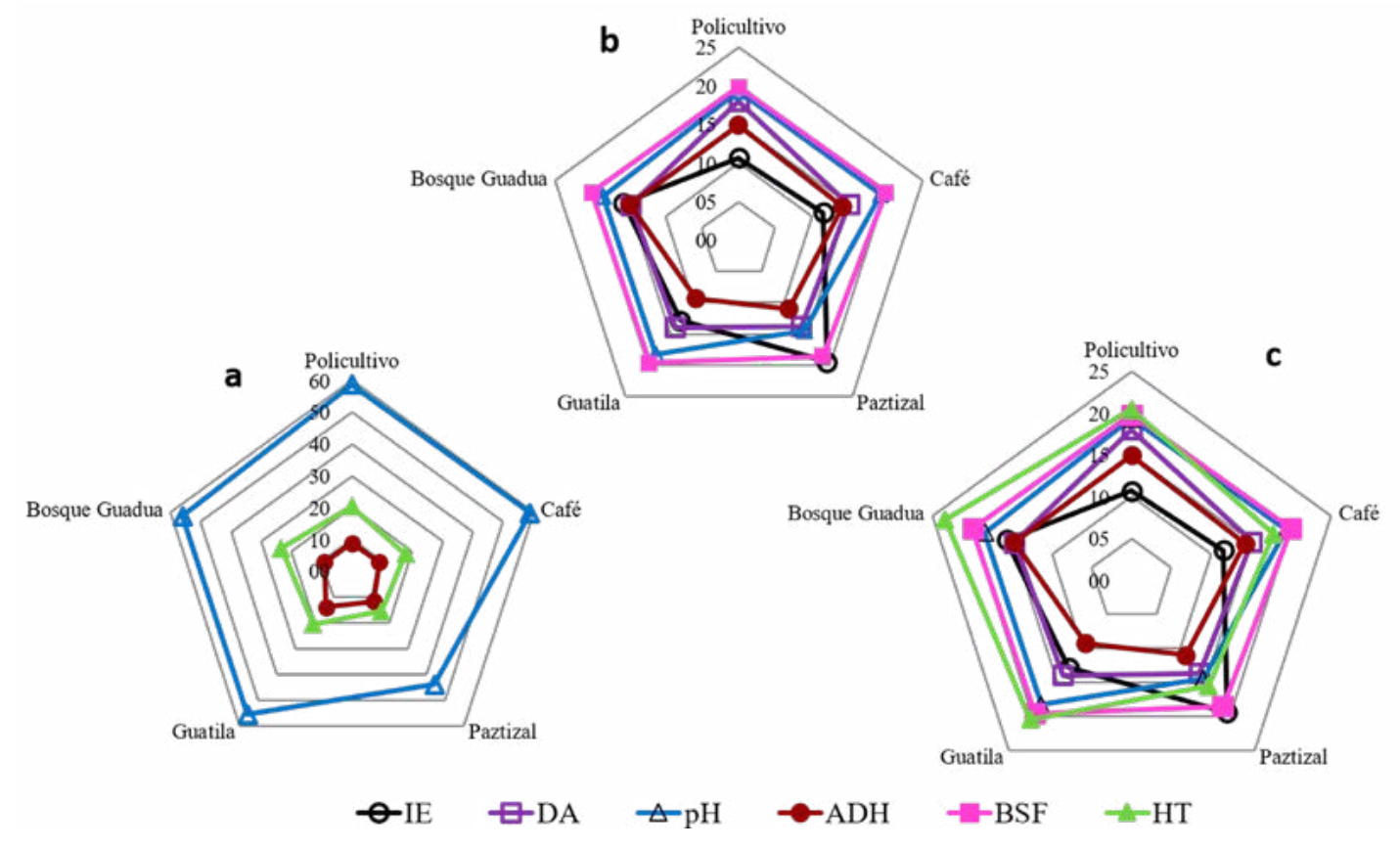

Figura 2. Contribución relativa (porcentaje) de los indicadores seleccionados en la determinación del índice de calidad de suelos para los CMD con tres (a), cinco (b) y seis indicadores (c) para los SP evaluados. IE= índice de estabilidad; $\mathrm{DA}=$ densidad aparente; $\mathrm{pH} ; \mathrm{ADH}=$ actividad deshidrogenasa; $\mathrm{BSF}=$ bacterias solubilizadoras de fosfato; $\mathrm{HT}=$ heterótrofos. 


\section{DISCUSIÓN}

Estudios previos han determinado índices de calidad edáfica en distintos agroecosistemas, para lo cual se han empleado un número variado de indicadores (en cuanto a número y tipo de indicadores). Se resalta que no hay estandarización ni homogenización para el proceso de selección del CMD, dado que los indicadores varían significativamente y dependen del contexto de la investigación, así como de factores inherentes al suelo y su manejo; $y$, finalmente, algunos no cumplen con todos los criterios que debe efectuar un indicador ideal de calidad de suelos (Andrews et al., 2002; Pulido et al., 2017; Vallejo, 2013).

Puglisi et al., (2006) calcularon tres índices en diferentes sitios agrícolas contaminados con desechos industriales y municipales usando como indicadores siete actividades enzimáticas. Sus resultados mostraron que el ICS constituido con tres indicadores ( $\beta$-glucosidasa, fosfatasa y ureasa) discriminó los suelos alterados de los controles con mayores puntuaciones, contrario a los otros ICS con siete y cuatro indicadores. Por su parte, Mishra et al., (2018) incluyeron cuatro variables fisicoquímicas al CMD para el cálculo del ICS en bosques tropicales caducifolios en India, siendo estas: la conductividad eléctrica, la densidad aparente, el $\mathrm{Mg}$ intercambiable y el $\mathrm{P}$ disponible. Similarmente, Zhang et al. (2016) conformaron su índice de calidad en suelos en humedales costeros en el delta del río amarillo en China a partir de cinco indicadores, siendo estos: el contenido de carbono orgánico, la conductividad eléctrica, el contenido de $\mathrm{Mg}$, la concentración de $\mathrm{N}-\mathrm{NO}_{3}$ y de azufre, provenientes de 13 propiedades totales del suelo. Liu et al. (2014) evaluaron la calidad del suelo en arroceras empleando un CMD que incluyó las siguientes variables: nitrógeno total, el $\mathrm{pH}$, contenido de Si y $\mathrm{Zn}$ disponible y el carbono de la biomasa microbiana, de 21 variables de suelos inicialmente valoradas. Finalmente, Nosrati (2013) evaluó la calidad del suelo en distintos usos del suelo y erosión en Irán, quienes seleccionaron nueve variables o indicadores para el cálculo del índice de calidad, siendo estas: el carbono orgáni$\mathrm{CO}$, nitrógeno total, actividad ureasa, la $\beta$-glucosidasa, fosfatasa alcalina, ADH, contenido de Mn, P y limo.

En el presente estudio se evidenció que los valores de los ICS fueron significativamente mayores cuando se usaron un menor número de indicadores (tres) en comparación con los CMD conformados por cinco o seis indicadores; lo que permitió, discriminar y evidenciar mejor las diferencias entre los agroecosistemas comparados. Este resultado es comparable con Askari y Holden (2014) quienes encontraron el mejor ICS calculado a partir de un CMD conformado por tres variables en su caso carbono orgánico $(\mathrm{C})$, relación de carbono y nitrógeno y la densidad aparente (DA) a partir de siete indicadores iniciales. Por su parte, Singh et al. (2014) enunciaron tres categorías principales para los ICS en categorías como baja, media y alta. En condiciones naturales los suelos de bosque y pastizales tienen una "alta" calidad con un ICS $>0.75$, mientras que agroecosistemas bajo manejo convencional corresponderían a una categoría "baja" con un ICS $<0.50$, y los aquellos manejos sostenibles recientemente convertidos o en proceso de reconversión podrían estar en una categoría "media" con un $0.50<\mathrm{ICS}<0.75$. Según esta clasificación, para esta investigación el PO, GA y CA representaron agroecosistemas de una alta calidad, siendo los ICS > 0.75. Por su parte, el PA y GU variaron entre una calidad media y baja dependiendo del tipo de CMD usado en el cálculo.

Estudios similares llevados a cabo por Rahmanipour et al. (2014), seleccionaron un CMD de cinco indicadores, partiendo de 10 variables; de manera similar, Yu et al. (2018) formaron un CMD de cuatro indicadores, de un conjunto de datos inicial de 13 propiedades. Nuestros resultados mostraron mayores valores de ICS para PO y CA, caracterizados por llevar a cabo prácticas agroecológicas, incluso con valores similares al GA. Estos resultados se comparan con los de Glover, Reganold y Andrews (2000), quienes evaluaron prácticas de 
manejo convencional, integrado y orgánico en las propiedades del suelo en huertos de manzanas, encontrando mayores valores de los ICS en sistemas con una labranza mínima. De igual forma, Lima et al. (2013), estudiaron diferentes sistemas de manejo para el arroz (labranza convencional, pregerminado y sin labranza) y encontraron los ICS más bajos en los sistemas convencionales, asociados a una menor actividad biológica.

Los bajos valores en el ICS del PA podrían estar relacionado con las actividades o el manejo al cual había sido sometido este agroecosistema, al ser un monocultivo, altamente dependiente de insumos químicos externos y del uso de maquinaria agrícola, lo cual se ha reportado previamente que genera un deterioro en la calidad del suelo (Vallejo et al., 2012; Askari y Holden, 2014; Vallejo et al., 2018). Por su parte, las diferencias entre el GU y PA podrían estar relacionadas con el periodo de descanso en el PA, lo cual favorece la recuperación de la estabilidad estructural e incrementa la actividad enzimática (Raiesi y Salek-Gilani, 2018).

El pH fue el indicador químico con mayor contribución en el cálculo del ICS para CMD con tres indicadores, mientras que los indicadores biológicos como BSF y HT fueron los de mayor contribución para cinco y seis indicadores respectivamente. El $\mathrm{pH}$ del suelo es una de las propiedades más importantes para determinar la calidad del suelo, dado que influye directamente en las reacciones químicas del suelo, en la disponibilidad de nutrientes y controla la diversidad y actividad de los microorganismos que desempeñan importantes funciones en el suelo (Zhijun et al., 2018). Así mismo, es una variable que responde a diferentes cambios en el manejo en distintos agroecosistemas, particularmente en aquellos que son dependientes del uso de fertilizantes químicos (Navas et al., 2011; Vallejo et al., 2012; Gutiérrez, Cardona y Monsalve, 2017). Así es como el pH ha sido incluido de forma habitual en los estudios y monitoreos de suelo y en las bases de datos existentes, siendo una propiedad de fácil determinación tanto en campo como en laboratorio; así mismo, el procedimiento o metodología es económico en comparación con otro tipo de indicadores químicos (Schoenholtz, Miegroet y Burger, 2000; Gutiérrez et al., 2017).

Por su parte, las propiedades biológicas y bioquímicas del suelo son más dinámicas y sensibles a las perturbaciones ambientales y a los cambios de manejo y uso del suelo, respondiendo rápidamente a dichos cambios; por ello, tienen la ventaja de servir como señales tempranas de degradación y pérdida de calidad edáfica (Vallejo et al., 2011). Las enzimas intervienen en la mayoría de los procesos que tienen lugar en el suelo y las funciones que realizan son de gran relevancia. Son responsables de la formación de moléculas orgánicas y particularmente tienen una participación vital en el ciclo nitrógeno, fósforo y carbono. Particularmente, la actividad de la deshidrogenasa permite, de manera global, tener una idea de los procesos microbianos que ocurren en el suelo debido a que se encuentran presentes únicamente en sistemas vivos, que indican, además, la tasa de oxidación de la materia orgánica. Es por esto que, debido a su relación con procesos de gran importancia en el suelo, la determinación de la actividad enzimática ha sido estudiada como un indicador de diferentes condiciones de calidad edáfica en agroecosistemas (Puglisi et al., 2006; Nosrati, 2013; Vallejo et al., 2018).

En contraste, la densidad de heterótrofos totales es otro indicador de gran relevancia en estudios de calidad de suelos, ya que responde rápidamente a cambios en la cantidad y tipo de residuos vegetales, al tipo de fertilización, química u orgánica, lo cual altera la disponibilidad de recursos energéticos requeridos para el crecimiento y actividad de estos microorganismos; así como a cambios con respecto a propiedades como el porcentaje de humedad, el porcentaje de $\mathrm{C}$ orgánico y $\mathrm{N}$ total y el $\mathrm{pH}$, las cuales se ven muy afectados en sistemas bajo manejo convencional (Vallejo et al., 2018).

Finalmente, la densidad de BSF es un indicador de gran relevancia pues aumentan la cantidad fósforo que, al ser hidrolizado con enzimas como las fitasas, facilitan la movilidad de este elemento 
en el suelo y lo transforman en un compuesto accesible para la planta. Razón por la cual constituyen una excelente alternativa para reducir la cantidad de fertilizantes aplicados a diferentes cultivos (Corrales, Arévalo y Moreno, 2014). De manera general, las prácticas agrícolas de conservación que involucran las rotaciones de cultivos, labranza cero, los cultivos de cobertura, el uso de abonos de animales, mantienen o aumentan la calidad del suelo al evidenciarse un incremento de la biodiversidad, particularmente, de muchos grupos funcionales microbianos (Cubillos et al., 2016; Ashworth et al., 2017).

\section{CONCLUSIONES}

Independiente del número de indicadores, los agroecosistemas con mayor calidad fueron el policultivo y los guaduales. La diferenciación entre agroecosistemas fue más evidente y significativa cuando se emplearon solo tres variables (conjunto mínimo de datos) en el índice de calidad de suelos. Las variables que se mantuvieron en los tres conjuntos mínimos de datos fueron: la densidad aparente, el índice de estabilidad, el pH, la actividad deshidrogenasa, la densidad de heterótrofos y bacterias solubilizadoras de fosfato. Estos indicadores son recomendables para ser utilizados en futuros estudios para evaluar la calidad de los suelos de la región, convirtiéndose en herramientas muy útiles para caracterizar y diagnosticar distintos manejos, tendientes a aumentar la productividad agrícola y a favorecer la sostenibilidad y la conservación del suelo. En este estudio se pudo evidenciar que un grupo de datos de tres, cinco o seis variables son suficientes para proporcionar información del estado general de los suelos en agroecosistemas, lo cual ayudará a en el establecimiento de sistemas de alerta y monitoreo al requerir un menor número de variables, reduciendo tiempo y costos de análisis.

\section{CONFLICTO DE INTERÉS}

Los autores declaran no tener conflicto de intereses.

\section{CONTRIBUCIÓN POR AUTOR}

Victoria Eugenia Vallejo Quintero y Laura Natali Afanador-Barajas idearon y lideraron la presente investigación. Victoria Eugenia Vallejo Quintero, Laura Natali Afanador-Barajas, Dainer Andrés Coca Peña, Andrés Felipe Vargas Giraldo, María Fernanda Bautista Murcia y Angélica Mendoza Hernández realizaron muestreos y mediciones en campo. Dainer Andrés Coca Peña, Andrés Felipe Vargas Giraldo, María Fernanda Bautista Murcia y Angélica Mendoza Hernández determinaron las características físicas, químicas y biológicas. Laura Natali Afanador-Barajas realizó los cálculos para la determinación de los ICS; y junto con Victoria Eugenia Vallejo Quintero realizaron la estadística y redactaron el manuscrito. Todos los autores contribuyeron a la discusión de los borradores.

\section{AGRADECIMIENTOS}

A la Universidad Central, Facultad de Ingeniería y Ciencias Básicas por la financiación del presente estudio (código No 20301119). Así mismo, agradecemos a la Congregación de Hermanas de la Caridad Dominicas de la Presentación, finca Nazareth, Vereda Petaluma, municipio de Cachipay.

\section{REFERENCIAS}

Andrews, S., Karlen, D. y Mitchell., J. (2002). A comparison of soil quality indexing methods for vegetable production systems in Northern California. Agriculture ecosystems \& environment, 90 (1), 25-45. https://doi.org/10.1016/S0167-8809(01)00174-8 
Ashworth, A., DeBruyn, J., Allen, F., Radosevich, M. y Owens, P. (2017). Microbial community structure is affected by cropping sequences and poultry litter under long-term no-tillage. Soil Biology Biochemistry, 114, 210-219.

https://doi.org/10.1016/j.soilbio.2017.07.019

Askari M. y Holden, N. (2014). Indices for quantitative evaluation of soil quality under grassland management. Geoderma, 230, 131-142.

https://doi.org/10.1016/j.geoderma.2014.04.019

Cantú, M., Becker, A., Bedano, J. y Schiavo, H. (2007). Evaluación de la calidad de suelos mediante el uso de indicadores e índices. Ciencia del Suelo, 25(2), 173178. Recuperado de

http://www.scielo.org.ar/scielo.php?script=sci_arttext\&pid=S1850-20672007000200008\&lng=es\&tlng=es

Casida, L., Klein, D. y Santoro, T. (1964). Soil dehydrogenase activity. Soil Science, 98(6), 371-376.

https://doi.org/10.1097/00010694-196412000-00004

Chen, Y., Wang, H., Zhou, J., Xing, L., Zhu, B., Zhao, Y. y Chen, X. (2013). Minimum Data Set for Assessing Soil Quality in Farmland of Northeast China. Pedosphere, 23(5), 564-576.

https://doi.org/10.1016/S1002-0160(13)60050-8

Corrales, L., Arévalo, Z. y Moreno, V. (2014). Solubilización de fosfatos: una función microbiana importante en el desarrollo vegetal. Nova, 12(21), 68-79. Recuperado de

http://www.scielo.org.co/scielo.php?script=sci_arttext\&pid=S1794-24702014000100006\&nrm=iso

Cubillos, A., Vallejo, V., Arbeli, Z., Terán, W., Dick, R., Molina, C., Molina, E. y Roldan, F. (2016). Effect of the conversion of conventional pasture to intensive silvopastoral systems on edaphic bacterial and ammonia oxidizer communities in Colombia. European Journal Soil Biology, 72, 42-50.

https://doi.org/10.1016/j.ejsobi.2015.12.003

Doran, J. y Parkin, T. (1994). Defining and assessing soil quality. En: J. W.Doran, D. C. Coleman, D. F. Bezdicek y B. A. Stewart (eds.), Defining Soil Quality for a Sustainable Environment (pp. 3-21). SSSA Special Publication Number 35.. Madison, Wisconsin, EE. UU.: Soil Science Society of America Inc.
EOT Cachopay (2008). Esquema de ordenamiento territorial Cachipay Cundinamarca 2008-2011. 417 p. Recuperado de

http://cdim.esap.edu.co/BancoConocimiento/C/cachipay_-_cundinamarca_-_eot_-_2008/cachipay_-_ cundinamarca_-_eot_-_2008.asp

Estrada-Herrera, I., Hidalgo-Moreno, C., Guzmán-Plazola, R., Almaraz Suárez, J., Navarro-Garza, H. y Etchevers-Barra, J. (2017). Indicadores de calidad de suelo para evaluar su fertilidad. Agrociencia, 51, 813-831. Recuperado de

http://www.scielo.org.mx/scielo.php?script=sci_arttext\&pid=S1405-31952017000800813\&nrm=iso

Fernandes, J., Gamero, C., Rodrigues, J. y Mirás-Avalos, J. (2011). Determination of the quality index of a Paleudult under sunflower culture and different management systems. Soil and Tillage Research, 112(2), 167-174.

https://doi.org/10.1016/j.still.2011.01.001

Flores, L. y Alcalá, J. (2010). Manual de procedimientos analíticos: laboratorio de física de suelos. Universidad Nacional Autónoma de México. Instituto de Geología. 56 p.

García, Y., Ramírez, W. y Sánchez, S. (2012). Indicadores de la calidad de los suelos: una nueva manera de evaluar este recurso. Pastos y Forrajes, 35(2), 125-138. Recuperado de

http://scielo.sld.cu/scielo.php?script=sci_arttext\&pid=S0864-03942012000200001\&lng=es\&tlng=es

Ghaemi, M., Astaraei, A., Emami, H., Nassiri-Mahalati, M. y Sanaeinejad, S. (2014). Determining soil indicators for soil sustainability assessment using principal component analysis of Astan Qudseast of Mashhad- Iran. Journal of Soil Science and Plant Nutrition, 14(4), 987-1004.

https://doi.org/10.4067/S0718-95162014005000077

Glover, J., Reganold, J.y Andrews, P. (2000). Systematic method for rating soil quality of conventional, organic, and integrated apple orchards in Washington State. Agriculture Ecosystems Environment, 80(1-2), 29-45.

https://doi.org/10.1016/S0167-8809(00)00131-6 
Goodfellow, M. y Williams, S. (1983). Ecology of actinomycetes. Annual Review of Microbiology, 37, 189-216.

\section{https://doi.org/10.1146/annurev.mi.37.100183.001201}

Guo, L., Sun, Z., Ouyang, Z., Han, D. y Li, F. (2017). A comparison of soil quality evaluation methods for Fluvisol along the lower Yellow River. Catena, 152, 135-143.

https://doi.org/10.1016/j.catena.2017.01.015

Gutiérrez, J., Cardona, Q. y Monsalve, O. (2017). Potencial en el uso de las propiedades químicas como indicadores de calidad de suelo. Una revisión. Revista Colombiana Ciencias Horticolas, 11(2), 450-458.

https://doi.org/10.17584/rcch.2017v11i2.5719

Hammer, Ø., Harper, D. y Ryan, P. (2012). PAST Paleontological Statistics version 2.16. Software package for education and data analysis. Paleontología Eletrónica, 4(1), 1-9. Recuperado de http://folk.uio. no/ohammer/past/

Karlen, D. y Stott, D. (1994). A framework for evaluating physical and chemical indicators of soil quality. En: J. W. Doran, D. C. Coleman, D. F. Bezdicek y B.A. Stewart, (eds.), Defining Soil Quality for a Sustainable Environment (pp. 53-72). Madison, SSSA.

Lê, S., Josse, J. y Husson, F. (2008). FactoMine R: An R Package for Multivariate Analysis. Journal of statistical software, 25(1), 1-18.

\section{https://doi.org/10.18637/jss.v025.i01}

Lima, A., Brussaard, L., Totola, M., Hoogmoed, W. y Goede, R. (2013). A functional evaluation of three indicators sets for assessing soil quality. Applied Soil Ecology, 64, 194-200.

https://doi.org/10.1016/j.apsoil.2012.12.009

Liu, Z., Zhou, W., Shen, J., Li, S., Liang, G., Wang, X., Sun, J. y Al, C. (2014). Soil quality assessment of acid sulfate paddy soils with different productivities in Guangdong Province, China. Journal of Integrative Agriculture, 13(1), 177-186.

https://doi.org/10.1016/S2095-3119(13)60594-8

Mandal, U., Warrington, D., Bhardwaj, A., Bar-Tal, A., Kautsky, L., Minz, D. y Levy, G. (2008). Evaluating impact of irrigation water quality on a calcareous clay soil using principal component analysis. Geoderma, 144(1-2), 189-197.

https://doi.org/10.1016/J.geoderma.2007.11.014

Masto, R., Chhonkar, P., Singh, D. y Patra, A. (2007). Soil quality response to long-term nutrient and crop management on a semi-arid Inceptisol. Agriculture Ecosystems \& Environment, 118(1-4), 130-142.

https://doi.org/10.1016/j.agee.2006.05.008

Mishra, G., Marzaioli, R., Giri, K. y Pandey, S. (2018). Soil quality assessment across different stands in tropical moist deciduous forests of Nagaland, India. Journal of Forestry Research, 1-7.

https://doi.org/10.1007/s11676-018-0720-8

Muñoz-Rojas, M. (2018). Soil quality indicators: critical tools in ecosystem restoration. Current Opinion in Environmental Science \& Health, 5, 47-52.

https://doi.org/10.1016/j.coesh.2018.04.007

Mursec, M., Leveque, L., Chaussod, R. y Curmi, P. (2018). The impact of drip irrigation on soil quality in sloping orchards developed on marl - a case study. Plant Soil Environment, 64, 20-25.

https://doi.org/10.17221/623/2017-pse

Nabiollahi, K., Taghizadeh-Mehrjardi, R., Kerry, R. y Moradian, S. (2017). Assessment of soil quality indices for salt-affected agricultural land in Kurdistan Province, Iran. Ecological Indicators, 83, 482-494.

https://doi.org/10.1016/j.ecolind.2017.08.001

Nautiyal, S. (1999). An efficient microbiological growth medium for screening phosphate solubilizing microorganisms. FEMS Microbiology Letters, 170(1), 265-270.

https://doi.org/10.1016/S0378-1097(98)00555-2

Navas, M., Benito, M., Rodríguez, I. y Masaguer, A. (2011). Effect of five forage legume covers on soil quality at the Eastern plains of Venezuela. Applied Soil Ecology, 49, 242-249.

https://doi.org/10.1016/j.apsoil.2011.04.017

Nosrati, K. (2013). Assessing soil quality indicator under different land use and soil erosion using multivariate statistical techniques. Environmental Monitoring and Assessment, 185(4), 2895-2907.

https://doi.org/10.1007/s10661-012-2758-y 
Obade, V. y Lal, R. (2016). Towards a standard technique for soil quality assessment. Geoderma, 265, 96-102.

https://doi.org/10.1016/j.geoderma.2015.11.023

Puglisi E., Del Re, A., Rao, M. y Gianfreda, L. (2006). Development and validation of numerical indexes integrating enzyme activities of soils. Soil Biology and Biochemistry, 38, 1673-1681.

https://doi.org/10.1016/j.soilbio.2005.11.021

Pulido, M., Schnabel, S., Contador, F., Lozano-Parra, J y Gómez-Gutiérrez, A. (2017). Selecting indicators for assessing soil quality and degradation in rangelands of Extremadura (SW Spain). Ecological Indicators, 74, 49-61.

https://doi.org/10.1016/j.ecolind.2016.11.016

R Development Core Team (2016). R: A Language and Environment for Statistical Computing. R Foundation for Statistical Computing. Vienna, Austria. Recuperado de

https://www.R-project.org/

Raiesi, F. y Salek-Gilani, S. (2018). The potential activity of soil extracellular enzymes as an indicator for ecological restoration of rangeland soils after agricultural abandonment. Applied Soil Ecology, 126, 140-147.

https://doi.org/10.1016/j.apsoil.2018.02.022

Rahmanipour, F., Marzaioli, R., Bahrami, H., Fereidouni, Z. y Bandarabadi, S. (2014). Assessment of soil quality indices in agricultural lands of Qazvin Province, Iran. Ecological Indicators, 40, 19-26.

https://doi.org/10.1016/j.ecolind.2013.12.003

Ramette, A. (2007). Multivariate analyses in microbial ecology. FEMS Microbiology Ecology, 62, 142-160. https://doi.org/10.1111/j.1574-6941.2007.00375.x

Rezaei, S., Gilkes, J. y Andrews, S. (2006). A minimum data set for assessing soil quality in rangelands. Geoderma, 136(1-2), 229-234.

https://doi.org/10.1016/j.geoderma.2006.03.021

Singh, A., Bordoloi, L., Kumar, M., Hazarika, S. y Parmar, B. (2014). Land use impact on soil quality in eastern Himalayan region of India. Environmental Monitoring Assessment, 186(4), 2013-2024.

https://doi.org/10.1007/s10661-013-3514-7
Schoenholtz, S., Miegroet, H. V. y Burger, J. (2000). A review of chemical and physical properties as indicators of forest soil quality: challenges and opportunities. Forest Ecology and Management, 138(1-3), 335-356.

https://doi.org/10.1016/S0378-1127(00)00423-0

Vallejo V. (2013). Importancia y utilidad de la evaluación de la calidad de suelos mediante el componente microbiano: experiencias en sistemas silvopastoriles. Colombia Forestal, 16(1), 83-99.

https://doi.org/10.14483/udistrital.jour.colomb. for.2013.1.a06

Vallejo, V., Gómez, M., Cubillos, A. y Roldán, F. (2011). Effect of land use on the density of nitrifying and denitrifying bacteria in the Colombian Coffee Region. Agronomía Colombiana, 29, 455-463. Recuperado de

http://www.scielo.org.co/scielo.php?script=sci_arttext\&pid=S0120-99652011000300015\&lng=es\&tlng=.

Vallejo, V., Arbeli, Z., Terán, W., Lorenz, N., Dick, R. y Roldan, F. (2012). Effect of land management and Prosopis juliflora (Sw.) DC trees on soil microbial community and enzymatic activities in intensive silvopastoral systems of Colombia. Agriculture Ecosystems \& Environment, 150, 139-148.

https://doi.org/10.1016/j.agee.2012.01.022

Vallejo, V., Afanador, L., Hernández, M. y Parra, D. (2018). Efecto de la implementación de diferentes sistemas agrícolas sobre la calidad del suelo en el municipio de Cachipay, Cundinamarca, Colombia. Bioagro, 30(1), 27-38. Recuperado de

http://www.ucla.edu.ve/bioagro/Rev30(1)/3.\%20 ms\%201718.pdf

Wilson, M. G. (2017). Manual de indicadores de calidad del suelo para las ecorregiones de Argentina. Buenos Aires: Ediciones INTA. p. 285. Recuperado de

https://inta.gob.ar/sites/default/files/manual_ics_final. pdf

Wymore, A. W. (1993). Model-Based Systems Engineering: An introduction to the mathematical theory of discrete systems and to the Tricotyledon theory of system design. Boca Raton, Florida, EE. UU.: CRC Press. 710 p. 
Yu, P., Liu, S., Zhang, L., Li, Q. y Zhou, D. (2018). Selecting the minimum data set and quantitative soil quality indexing of alkaline soils under different land uses in northeastern China. Science of the Total Environment, 616-617, 564-571.

https://doi.org/10.1016/j.scitotenv.2017.10.301

Zhang, G., Bai, J., Xi, M., Zhao Q., Lu, Q. y Jia, J. (2016). Soil quality assessment of coastal wetlands in the Yellow River Delta of China based on the minimum data set. Ecological Indicators, 66, 458-466.

https://doi.org/10.1016/j.ecolind.2016.01.046
Zhijun, H., Selvalakshmi, S., Vasu, D., Liu, Q., Cheng, H., Guo, F. y Ma, X. (2018). Identification of indicators for evaluating and monitoring the effects of Chinese fir monoculture plantations on soil quality. Ecological Indicators, 93, 547-554.

https://doi.org/10.1016/j.ecolind.2018.05.034

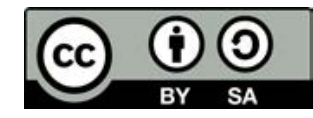

Colombia Forestal •ISSN 0120-0739 • e-ISSN 2256-201X • Bogotá-Colombia • Vol. 23 No. 1 • Enero-Junio de 2020 •pp. 35-50 